\title{
Genetic methods to identify and manipulate newly born neurons in the adult brain
}

\author{
Itaru Imayoshi' ${ }^{1,2,3}$, Masayuki Sakamoto ${ }^{1,4,5}$ and Ryoichiro Kageyama ${ }^{1,4 *}$ \\ 1 Institute for Virus Research, Kyoto University, Kyoto, Japan \\ 2 The Hakubi Center, Kyoto University, Kyoto, Japan \\ 3 Japan Science and Technology Agency, Precursory Research for Embryonic Science and Technology, Japan \\ 4 Japan Science and Technology Agency, Core Research for Evolutional Science and Technology, Japan \\ ${ }^{5}$ Graduate School of Biostudies, Kyoto University, Kyoto, Japan
}

\section{Edited by:}

Silvia De Marchis, University of Turin, Italy

\section{Reviewed by:}

Claudio Giachino, Max Planck Institute Germany

Michele Studer, INSERM, France

\section{${ }^{*}$ Correspondence:}

Itaru Imayoshi and Ryoichiro

Kageyama, Institute for Virus Research,

Kyoto University, Shogoin-Kawahara

Sakyo-ku, Kyoto 606-8507, Japan.

e-mail: iimayosh@virus.kyoto-u.ac.jp;

rkageyam@virus.kyoto-u.ac.jp
Although mammalian neurogenesis is mostly completed by the perinatal period, new neurons are continuously generated in the subventricular zone of the lateral ventricle and the subgranular zone of the hippocampal dentate gyrus. Since the discovery of adult neurogenesis, many extensive studies have been performed on various aspects of adult neurogenesis, including proliferation and fate-specification of adult neural stem cells, and the migration, maturation and synaptic integration of newly born neurons. Furthermore, recent research has shed light on the intensive contribution of adult neurogenesis to olfactory-related and hippocampus-mediated brain functions. The field of adult neurogenesis progressed tremendously thanks to technical advances that facilitate the identification and selective manipulation of newly born neurons among billions of pre-existing neurons in the adult central nervous system. In this review, we introduce recent advances in the methodologies for visualizing newly generated neurons and manipulating neurogenesis in the adult brain. Particularly, the application of site-specific recombinases and Tet inducible system in combination with transgenic or gene targeting strategy is discussed in further detail.

Keywords: adult neurogenesis, neural stem cells, Cre/loxP, CreERT2, transgenic mice, nestin

\section{INTRODUCTION}

It is now widely accepted that in mammals, including humans, newly born neurons are continuously generated and incorporated into the functional neural network of the adult brain (McKay, 1997; Gage, 2000; Temple, 2001; Ming and Song, 2005; Imayoshi et al., 2008, 2009). Neurogenesis in the mature adult brain was first reported by Altman and colleagues using a $\left[\mathrm{H}^{3}\right]$-thymidine-incorporation labeling method in the dentate gyrus (DG) of the rat hippocampus (Altman and Das, 1965). They published a series of research articles describing neurogenesis in various regions of the adult rat brain, including the neocortex and olfactory bulb (OB; Altman, 1966, 1969). The long-term survival of newly born neurons in the hippocampus has also been demonstrated (Kaplan and Hinds, 1977). The development of a bromodeoxyuridine (BrdU)-incorporation labeling method enabled us to analyze the characteristics of newly born neurons in combination with immunohistochemistry. Adult neurogenesis has been observed with BrdU incorporation in all mammals examined, including humans (Eriksson et al., 1998).

In the rodent brain, neurogenesis predominantly occurs in two brain regions, the subventricular zone (SVZ) of the lateral ventricles and the subgranular zone (SGZ) of the hippocampal DG (Alvarez-Buylla et al., 2001; Lledo et al., 2006; Zhao et al., 2006). A large number of neurons born in the SVZ migrate into the OB and become local interneurons (granule cells and periglomerular cells), while neurons born in the SGZ migrate into the granule cell layer and become granule cells of the DG. Newly born neurons are incorporated into the functional neural networks of the $\mathrm{OB}$ and the DG, suggesting a significant impact of adult neurogenesis on neural circuit plasticity, and various brain functions, including learning and memory (van Praag et al., 2002; Carleton et al., 2003; Kee et al. 2007; Adam and Mizrahi, 2010; Deng et al., 2010; Lazarini and Lledo, 2011). Neurogenesis outside these two regions appears to be extremely limited in the intact adult mammalian central nervous system (CNS). After pathological stimulation, such as brain insults or seizures, adult neurogenesis appears to occur in regions otherwise considered to be non-neurogenic, such as the striatum and neocortex (Ming and Song, 2005; Gould, 2007).

The field of adult neurogenesis progressed thanks to technical advances that facilitate the identification and selective manipulation of newly born neurons among billions of pre-existing neurons in the adult CNS (Deng et al., 2010; Kelsch et al., 2010; Lacar et al., 2010). Newly generated neurons can be identified and/or manipulated by various approaches, such as the incorporation of nucleotide analogs, retrovirus-mediated gene transfer, and genetic methods using transgenic mice. The ablation of neural stem/progenitor cells using irradiation, with antimitotic drugs, or the transgenic expression of toxin genes are common strategies to study the functional importance of adult neurogenesis. Accumulating evidence has suggested a correlation between the magnitude of adult neurogenesis and an animal's cognitive ability. In this review, we discuss the experimental approaches to visualize newly generated neurons and manipulate neurogenesis in the adult brain, especially focusing on genetic methods. 


\section{NEUROGENESIS FROM NEURAL STEM CELLS IN THE ADULT BRAIN}

In the adult brain, neural stem cells (NSCs) exist principally in two regions: the SVZ of the lateral ventricle and the SGZ of the hippocampal DG (Kriegstein and Alvarez-Buylla, 2009), where neurogenesis occurs continuously. The SVZ is a layer extending along the lateral wall of the lateral ventricle and contains many dividing cells. A large number of neurons born in the SVZ migrate into the $\mathrm{OB}$, forming a rostral migratory stream, and differentiate into local interneurons. A subset of glial fibrillary acidic protein (GFAP)-positive cells (Type-B cells) function as NSCs in the adult SVZ. Type-B cells divide slowly and give rise to rapidly proliferating "transit-amplifying cells" (Type-C cells), which then generate migrating neuroblasts (Type-A cells) after several cell divisions. Type-B cells have the ultrastructural features of astrocytes and express GFAP, a canonical astrocyte marker protein (Doetsch et al., 1997, 1999; Pastrana et al., 2009; Figure 1A). Thus, NSCs gradually undergo changes in proliferation and differentiation competencies in the developing and adult brain (Kriegstein and Alvarez-Buylla, 2009).

In the SGZ of the adult hippocampal DG, Type-1 NSCs have astrocytic features and are marked by GFAP (Seri et al., 2004; Zhao et al., 2006; Suh et al., 2007). Although these cells have a proliferative capacity, they cycle much slower than the Type-2 progenitor cells that follow. While Nestin, Sox2, and brain lipidbinding protein (BLBP) are also expressed in Type- 1 cells, the expression persists into the Type-2 cell stages (Steiner et al., 2006). NeuroD and Doublecortin (Dcx) appear in Type-2b cells,
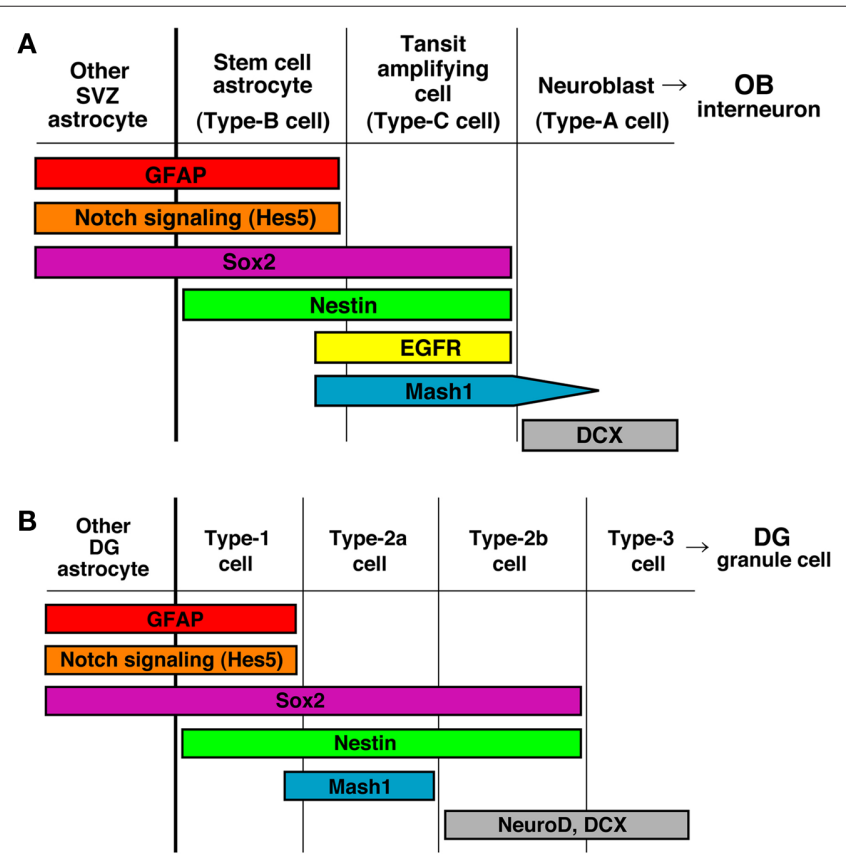

FIGURE 1 | Current view of the sequence of neurogenesis from NSCs in the adult brain. (A) Generation of new interneurons in the olfactory bulb (OB) from NSCs in the subventricular zone of the lateral ventricle (SVZ/LV). (B) Generation of new granular neurons in the dentate gyrus (DG) of the hippocampus from NSCs in the subgranular zone (SGZ). the later stage of Type- 2 cells, and persist into postmitotic but immature granule cell precursors. Finally, these cells mature into NeuN/calbindin/Prox1-positive granule cell neurons in the DG (Figure 1B). Notch signaling is highly activated in Type-B cells of the SVZ of the lateral ventricle and Type- 1 cells of the SGZ of the DG (Ehm et al., 2010; Imayoshi et al., 2010; Lugert et al., 2010; Figure 1).

\section{CreER TRANSGENIC MICE TO MANIPULATE ADULT NEURAL STEM/PROGENITOR CELLS}

Site-specific recombinases (SSRs) have proven to be useful tools in the analysis of gene function and the genetic manipulation of restricted cell populations (Branda and Dymecki, 2004). SSRs can induce the deletion, insertion or inversion of DNA sequences by breaking and joining DNA molecules at specific sites (Nagy, 2000; Wu et al., 2007). The most widely used SSR in cultured mammalian cells and animals is the P1 bacteriophage-derived Cre, a member of the $\lambda$ integrase family that recognizes homotypic 34-bp loxP recognition sites. Cre recombinase efficiently excises DNA, which is flanked by two directly repeated loxP recognition sites, in mammalian cells. By crossing transgenic mice expressing Cre in a cell type-specific manner with reporter mice, we can trace the lineage of the progeny of Cre expressing cells with reporter gene expression such as GFP and LacZ. In reporter mice, a reporter gene is under the control of a ubiquitous promoter such as CAG (a combination of the chicken beta-actin promoter and the cytomegalovirus immediate-early enhancer) and the Rosa 26 promoter; however the expression of the reporter gene is interrupted by a loxP-flanked transcriptional STOP cassette. In these mice, recombination by Cre results in the removal of the STOP cassette and permanent expression of the reporter protein.

Temporal control of Cre-mediated recombination can be achieved by using the ligand-dependent chimeric recombinase CreER. CreER is constructed by fusing Cre to the mutated ligandbinding domain (LBD) of the estrogen receptor (Metzger et al., 1995; Feil et al., 1997). In transgenic mice, CreER is activated by the administration of tamoxifen, a synthetic estrogen antagonist, but not by natural ligands of LBD such as $17 \beta$-estradiol (Indra et al., 2000; Li et al., 2000).

Several groups have generated transgenic mice using the nestin promoter and enhancer (Table 1). Nestin is an intermediate filament protein specifically expressed by neural stem/progenitor cells in the developing nervous system and the adult brain (Lendahl et al., 1990), and the second intron of the nestin gene contains a neural stem/progenitor cell-specific enhancer (Zimmerman et al., 1994; Mignone et al., 2004; Imayoshi et al., 2006, 2008; Lagace et al., 2007). In Nestin-CreER transgenic embryos, CreER is specifically expressed in the ventricular zone of the developing CNS and, in mice expressing CreER at an appropriate level, Cre recombinase activity is efficiently induced in NSCs by the administration of tamoxifen. Nestin is expressed in the SVZ and SGZ of the adult brain (Doetsch et al., 1997), and in Nestin-CreER transgenic mice, NSCs and transit-amplifying cells express CreER. In the presence of tamoxifen, Cre-mediated recombination occurs efficiently in NSCs, and the majority of newborn neurons generated from such recombined NSCs were labeled with reporter gene expression after tamoxifen treatment (Carlén et al., 2006; Imayoshi et al., 2006, 2008; 
Table 1 | CreER-expressing mice for Cre/loxP gene targeting in adult neurogenesis research.

\begin{tabular}{|c|c|c|c|}
\hline Promoter/transcription unit & CreER variant & Technique & Reference \\
\hline Nestin promoter +2 nd intron enhancer & CreERT2 & $\operatorname{Tg}$ & Imayoshi et al. $(2006,2008)$ \\
\hline Nestin promoter + Nestin exons 1-3 including the 2 nd intron & CreERT2 & $\operatorname{Tg}$ & Lagace et al. (2007) \\
\hline Nestin 2nd intron/hsp68 mini promoter & CreERT2 & $\operatorname{Tg}$ & Carlén et al. (2006) \\
\hline Nestin promoter + Nestin exons 1-3 including the 2 nd intron & CreERT2 & $\operatorname{Tg}$ & Giachino and Taylor (2009) \\
\hline Nestin promoter +2 nd intron enhancer & CreERT2 & $\operatorname{Tg}$ & Chen et al. (2009) \\
\hline Sox2 5 ' telencephalic enhancer/promoter & CreERT2 & $\operatorname{Tg}$ & Favaro et al. (2009) \\
\hline TLX BAC & CreERT2 & $\operatorname{Tg}$ & Liu et al. (2008) \\
\hline Gli1 locus & CreERT2 & $\mathrm{KI}$ & Ahn and Joyner (2005) \\
\hline FGFR3 PAC & iCreERT2 & $\operatorname{Tg}$ & Young et al. (2010) \\
\hline C $\times 30 \mathrm{BAC}$ & CreERT2 & $\operatorname{Tg}$ & Slezak et al. (2007) \\
\hline Aqp4 BAC & CreERT2 & $\operatorname{Tg}$ & Slezak et al. (2007) \\
\hline Ascl1 BAC & CreERTM & $\operatorname{Tg}$ & Kim et al. (2007) \\
\hline DIx1/2 intergenic enhancer 112 & CreERT2 & $\operatorname{Tg}$ & Batista-Brito et al. (2008) \\
\hline DCX BAC & CreERT2 & $\operatorname{Tg}$ & Cheng et al. (2010) \\
\hline DCX promoter & CreERT2 & $\operatorname{Tg}$ & Zhang et al. (2010) \\
\hline
\end{tabular}

BAC, bacterial artificial chromosome; PAC, phage artificial chromosome; Tg, transgenic; KI, knock-in.

Kuo et al., 2006; Balordi and Fishell, 2007; Burns et al., 2007; Lagace et al., 2007; Chen et al., 2009; Giachino and Taylor, 2009). Although the nestin promoter/enhancer is highly active in NSCs in the adult brain, undesired transgene expression, such as in ependymal cells and specific neuronal subtypes, was observed. Using other promoter sequences or gene loci of NSC-specific genes, for example Sox2, Tlx, Gli1, and FGFR3, several CreER driver transgenic mouse strains have been reported and efficient tamoxifen-dependent recombination was observed (Table 1); however, undesired transgene expression, especially in specific neuronal subtypes was also observed (Ahn and Joyner, 2005; Liu et al., 2008; Favaro et al., 2009; Young et al., 2010).

In CreER transgenic mice in which the expression of the transgene is regulated by an astrocyte-specific gene, adult neurogenesis can be targeted, because adult NSCs express astrocyte-specific genes, such as GFAP, GLAST, Cx30, and aquaporin4 (Table 1; Ganat et al., 2006; Mori et al., 2006; Slezak et al., 2007; Chow et al., 2008). In these transgenic mice, efficient recombination was observed in adult NSCs in a tamoxifen-dependent manner, but one concern is that recombination is also induced in all astrocytes, including non-neurogenic cells, and it may affect the behavior of NSCs in a non-cell-autonomous manner.

To achieve efficiently inducible CreER transgenic mice, it is important to identify a founder line in which CreER expression is optimal. Most adult neural stem/progenitor specific promoter sequences also induce transgene expression in NSCs during development, and in many cases their promoter activities are rather stronger in embryonic NSCs. In the absence of tamoxifen, CreER is trapped in the cytoplasm, but when CreER is expressed too much, CreER is not retained in the cytoplasm and significant amounts translocate into the nucleus, resulting in tamoxifen-independent recombination. Therefore, too-high level of CreER expression results in tamoxifen-independent recombination in NSCs during development and disturb adult NSC-specificity (Imayoshi et al., 2006; Chen et al., 2009). Conversely, too-low level of CreER expression impairs efficient tamoxifen-dependent recombination and induce recombination only in a subset of adult NSCs. The expression level and spatial pattern of transgenes could be affected by the transgene-copy number and chromosomal integration site. In the case of CreER knock-in mice, the expression level of CreER is determined by the activity of the original promoter from the targeted locus; therefore, we have to carefully select the targeting gene locus.

Various inducible CreER variants have been reported, among them, from our experience, CreER ${ }^{\mathrm{T} 2}$ developed in the Chambon laboratory is the most superior with respect to its induction by tamoxifen (Feil et al., 1997). $\mathrm{ER}^{\mathrm{T} 2} \mathrm{CreER}^{\mathrm{T} 2}$, in which $\mathrm{ER}^{\mathrm{T} 2}$ is fused to the $\mathrm{N}$ - and C-terminus of Cre (Casanova et al., 2002), has more tightly controlled inducibility than $\mathrm{CreER}^{\mathrm{T} 2}$, tamoxifen-independent leaky recombination is greatly reduced in the $\mathrm{ER}^{\mathrm{T} 2} \mathrm{CreER}^{\mathrm{T} 2}$ variant, but higher expression levels than for $\mathrm{CreER}^{\mathrm{T} 2}$ are required for efficient recombination (Casanova et al., 2002; Matsuda and Cepko, 2007). $\mathrm{ER}^{\mathrm{T} 2} \mathrm{CreER}{ }^{\mathrm{T} 2}$ is more suitable than CreER ${ }^{\mathrm{T} 2}$ for transgenic constructs whose promoter activities are very strong, or for fatemapping or lineage-trace experiments that require strictly specific recombination in adult NSCs. 
For the activation of CreER by tamoxifen or its metabolite 4-hydroxy-tamoxifen (4-OHT), intraperitoneal (I.P.) injection or oral gavage are commonly used. When high dose (e.g., $10 \mathrm{mg}$ per $30 \mathrm{~g}$ mouse weigh) tamoxifen treatment is required, I.P. injection is sometimes detrimental to the health of the recipient mouse, especially if tamoxifen is dissolved in corn oil. The effects of tamoxifen treatment itself on mouse health should be minimal, and, from our experience, oral gavage is less harmful to health than I.P. injection for high doses of tamoxifen.

\section{VISUALIZATION OF NEUROGENESIS}

Transgenic mice expressing CreER in adult NSCs are crossed with reporter mouse strains containing a floxed STOP sequence upstream of GFP or LacZ as a read out for recombinase activity (Table 2; Branda and Dymecki, 2004). In double transgenic mice, in the presence of tamoxifen, CreER is released from its cytoplasmic sequestration and translocated to the nucleus (activated). The Cre reporter allele undergoes recombination in cells expressing activated CreER, resulting in permanent marker (GFP or LacZ) expression in marked NSCs and their descendants (Metzger et al., 1995).

The Rosa26-stop-LacZ knock-in reporter mouse has most commonly been used (Soriano, 1999). As the Rosa26 locus is active in almost all cells, the Rosa 26 promoter has been used to drive widespread expression of marker genes in transgenic mice (Zambrowicz et al., 1997). Although X-gal staining to detect LacZ expression has very strong sensitivity and a low background signal, immunostaining for LacZ protein is occasionally difficult. As LacZ localizes in the cell body, it is difficult to clearly visualize cellular shapes, such as the axons or dendrites of neurons. GFP or its variants spread more diffusely than LacZ throughout cells, and fine cellular structures are more easily visualized during immunostaining for GFP than for LacZ. Rosa26-stop-ECFP/EGFP/EYFP knock-in reporter strains were developed and widely used in double- or triple-color immunostaining with various antibodies (Mao et al., 2001; Srinivas et al., 2001).

Using the ubiquitous CAG promoter, several transgenic Cre reporter strains have been developed (Table 2; Lobe et al., 1999; Kawamoto et al., 2000; Novak et al., 2000; Vintersten et al., 2004). Although CAG promoter transgenic mice are able to induce higher levels of reporter expression than the Rosa26 promoter in most cells, the promoter activity is silenced in some cells and not completely ubiquitous probably due to unfavorable chromatin configurations. To overcome this problem, several reporter strains were recently developed in which reporter constructs under the control of the CAG promoter were knocked into the Rosa26 locus. The expression of exogenous promoters inserted into this locus is not restricted by unfavorable chromatin configurations (Strathdee et al., 2006). Rosa26-knock-in Cre reporter strains with the CAG promoter have stronger reporter expression than the normal Rosa26 reporter and, additionally a single copy is introduced thereby avoiding problems associated with a large multicopy array. One of these reporter mice, the Rosa26-mTmG reporter (Muzumdar et al., 2007), induces the membrane-bound expression of EGFP after recombination. In this reporter mouse, fine cellular structures, especially axons of neurons, could be clearly visualized by its strong EGFP expression in the plasma membrane. Recently, Rosa26 knock-in Cre reporter

Table 2 | Site-specific recombinases (SSRs)-responsive effector mice.

\begin{tabular}{|c|c|c|c|c|}
\hline Promoter/transcription unit & Technique & STOP cassette & Reporter/effector & Reference \\
\hline Rosa26 locus & $\mathrm{KI}$ & loxP-flanked & LacZ & Soriano (1999) \\
\hline Rosa26 locus & $\mathrm{KI}$ & loxP-flanked & ECFP & Srinivas et al. (2001) \\
\hline Rosa26 locus & $\mathrm{KI}$ & loxP-flanked & EYFP & Srinivas et al. (2001) \\
\hline Rosa26 locus & $\mathrm{KI}$ & loxP-flanked & EGFP & Mao et al. (2001) \\
\hline CAG & $\operatorname{Tg}$ & loxP-flanked & PLAP & Lobe et al. (1999) \\
\hline CAG & $\operatorname{Tg}$ & loxP-flanked & EGFP & Novak et al. (2000) \\
\hline CAG & $\operatorname{Tg}$ & loxP-flanked & DsRed & Vintersten et al. (2004) \\
\hline CAG & $\operatorname{Tg}$ & loxP-flanked & EGFP & Kawamoto et al. (2000) \\
\hline CAG (Rosa26 locus) & $\mathrm{KI}$ & loxP-flanked & mEGFP & Muzumdar et al. (2007) \\
\hline Rosa26 locus + CAG & $\mathrm{KI}$ & loxP-flanked & tdTomato & Madisen et al. (2010) \\
\hline Tau locus & $\mathrm{KI}$ & loxP-flanked & mEGFP & Hippenmeyer et al. (2005) \\
\hline Rosa26 locus & $\mathrm{KI}$ & loxP-flanked & DTA & Brockschnieder et al. (2006) \\
\hline Rosa26 locus & $\mathrm{KI}$ & loxP-flanked & diphtheria toxin receptor & Buch et al. (2005) \\
\hline Rosa26 locus & $\mathrm{KI}$ & FRT-flanked & PLAP & Awatramani et al. (2001) \\
\hline Rosa26 locus & $\mathrm{KI}$ & FRT-flanked & LacZ & Possemato et al. (2002) \\
\hline Rosa26 locus & $\mathrm{KI}$ & attP/B-flanked & LacZ & Raymond and Soriano (2007) \\
\hline Rosa26 locus & $\mathrm{Kl}$ & rox-flanked & LacZ & Anastassiadis et al. (2009) \\
\hline Rosa26 locus & $\mathrm{KI}$ & loxP-flanked and FRT-flanked & PLAP & Awatramani et al. (2003) \\
\hline Rosa26 locus + CAG & $\mathrm{KI}$ & loxP-flanked and FRT-flanked & WGA-ires-EGFP & Farago et al. (2006) \\
\hline Rosa26 locus + CAG & $\mathrm{Kl}$ & loxP-flanked and FRT-flanked & EGFP & $\begin{array}{l}\text { Sousa et al. (2009), } \\
\text { Miyoshi and Fishell (2006) }\end{array}$ \\
\hline Rosa26 locus + CAG & $\mathrm{KI}$ & loxP-flanked and FRT-flanked & tetanus toxin light chain & Kim et al. (2009) \\
\hline
\end{tabular}

Tg, transgenic; KI, knock-in. 
strain series with the CAG promoter were reported by the Allen institute (Madisen et al., 2010). Among them, the Rosa26-CAGstop-tdTomato reporter strain is very useful in live imaging experiments, because tdTomato is the brightest available red fluorescent protein and is excited with a $543 \mathrm{~nm}$ laser, which is less toxic for cells than a $488 \mathrm{~nm}$ laser.

Endogenous Rosa26 promoter activity is very low in astrocytes compared with other neural cell types (Garcia et al., 2010), and the expression of the reporter protein is often undetectable in astrocytes. Unfortunately, this may lead to severe misunderstandings in fate-mapping experiments. As the CAG promoter is active in astrocytes, Rosa 26 knock-in reporter strains with the $\mathrm{CAG}$ promoter are more suitable for fate-mapping experiments.

In some cases, reporter expression in other cell types (e.g., astrocytes, oligodendrocytes, and NSCs) disturbs the observation of fine structures of marked newly born neurons. Several Cre reporter strains have been developed in which reporter protein expression is driven by neuron-specific promoters. One of these reporter mice, the Tau-stop-mGFP knock-in mouse, can induce the membranebound expression of EGFP driven by the endogenous neuronspecific Tau promoter, which is very useful for the visualization of axon targeting by newborn neurons (Hippenmeyer et al., 2005). However, it is important to check in advance that the Tau promoter is active in the newly born neurons of interest.

Several groups have been developed CreER driver strains in which CreER is expressed in transit-amplifying cells and neuroblasts, but not in NSCs (Table 1). In the Ascl1-CreER and Dlx1/2CreER lines, after the administration of tamoxifen, Cre-mediated recombination is specifically induced in transit-amplifying cells (Kim et al., 2007; Batista-Brito et al., 2008). The DCX-CreER line is able to induce recombination in immature newborn neurons (Cheng et al., 2010; Zhang et al., 2010). Importantly, as CreER is not expressed in NSCs, these lines allow pulse labeling of newborn neurons within a short time-window, thereby facilitating the analysis of the differentiation and integration of adult-born new neurons at specific time points.

Recently the Lois group and Mizrahi group reported elegant experimental methods for the genetic labeling of the synapses of adult-born neurons (Kelsch et al., 2008, 2009; Livneh et al., 2009). The visualization of pre- and post-synaptic terminals can be achieved by the expression of fluorescent proteins fused to proteins specifically located in synapses. For instance, synaptophysin is a protein localized in neurotransmitter vesicles that is selectively located at presynaptic terminals and can be used to identify release sites on axon terminals. PSD95, a scaffolding protein that is restricted to clusters in the post-synaptic density of most glutamatergic synapses, has been used to identify post-synaptic terminals. To achieve the sparse and modest expression of these fusion proteins, they adapted retrovirus-mediated gene transfer to adult NSCs.

Relatively little is known about the connectivity of newborn neurons within mature circuits of the adult brain (Deng et al., 2010). How new neurons make synaptic connections with mature circuits is an open question. Several genetically encoded synaptic tracers are used to characterize the connections between neurons. Genetically encoded tracer molecules, such as wheat germ agglutinin (WGA) or tetanus toxin- fragment C (TTC), can be transported trans-synaptically (Luo et al., 2008). Fusion proteins of these tracer molecules and GFP, LacZ, alkaline phosphatase, or Cre can also be transported trans-synaptically (Luo et al., 2008). The application of these genetically encoded synaptic tracers, or recently developed monosynaptic rabies virus technology (Wickersham et al., 2007; Miyamichi et al., 2011), may elucidate the connectivity of newly born neurons in neuronal circuitry and contribute to a functional understanding of the lifelong addition of new neurons in the adult brain.

\section{GENETIC MANIPULATION OF ADULT NEUROGENESIS}

Several methods have been developed to manipulate neurogenesis in the adult brain. The majority of studies have examined the consequences of the suppression of neurogenesis on cognitive performance (Deng et al., 2010). The traditional approaches for ablating neurogenesis are anti-mitotic drug treatments or irradiation. These treatments are able to selectively disrupt cell cycle progression in neural stem/progenitor cells, because these cells are more sensitive to these treatments than differentiated neurons. After treatment, neurogenesis is strongly though not fully blocked (Wojtowicz, 2006; Gould, 2007). Although these methods are effective in reducing neurogenesis, they have considerable side effects in animals, such as general health deterioration and inflammation.

Recently, several transgenic mouse lines have been generated for more specific suppression of adult neurogenesis. In GFAP-TK and Nestin-TK mice, the targeted expression of herpes simplex virus thymidine kinase (HSV-TK) in NSCs is combined with the delivery of the nucleotide analog ganciclovir (GCV), resulting in specific and inducible ablation of dividing GFAP- or Nestin-expressing cells (Figure 2A; Garcia et al., 2004; Saxe et al., 2006; Deng et al., 2009; Singer et al., 2009). In both transgenic mice, adult neurogenesis was severely reduced after GCV treatment. Although other proliferating populations expressing GFAP or Nestin could be affected throughout the body, these transgenic strategies are highly specific. Theoretically, this model affects dividing NSCs, and quiescent NSCs should not be affected, which allows investigators to transiently reduce neurogenesis, and after the cessation of GCV infusion, active neurogenesis resumes. Although it was reported that the recovery of neurogenesis after drug withdrawal, was slow and incomplete, possibly because of quiescent NSC population was also affected by the prolonged infusion of GCV, these transgenic models are very useful as temporally regulated and reversible methods for the manipulation of adult NSCs.

In the Nestin-CreER/NSE-DTA mouse (Imayoshi et al., 2008), the expression of a suicide gene (diphtheria toxin fragment A, DTA) is selectively induced in newly generated neurons. NSE-DTA mice carry the loxP-STOP-loxP-IRES-DTA gene cassette, which was knocked into the $3^{\prime}$-non-coding region of the neuron-specific enolase (NSE) gene (Figure 2B). Crossing NSE-DTA mice with Nes-CreER mice and administering tamoxifen led to the deletion of the STOP cassette in NSCs. When these NSCs began neuronal differentiation, DTA was expressed from the NSE locus, thereby killing the cells.

The induction of apoptotic cell death in NSCs was achieved in Nestin-rtTA/TRE-Bax mice (Figure 2C; Dupret et al., 2008). This model is based on the reverse tetracycline-controlled transactivator (rtTA)-regulated system (Tet-On system). The activation of transgenes with doxycycline (Dox) treatment, a tetracycline analog, 


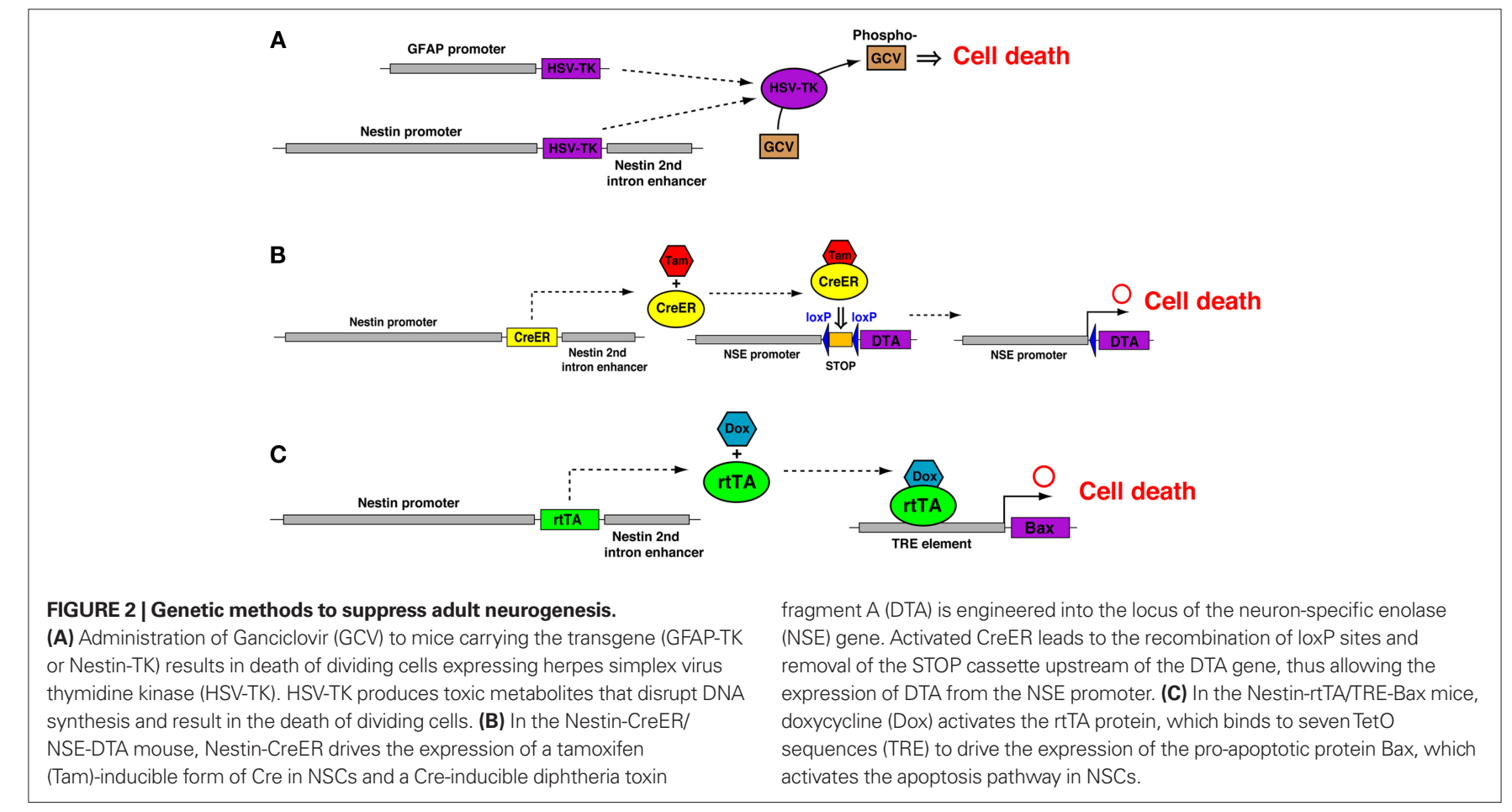

resulted in the overexpression of the pro-apoptotic protein Bax in nestin-expressing cells. This resulted in the increased cell death of neural stem/progenitor cells, consequently cell proliferation and neurogenesis were reduced, albeit not totally blocked.

In addition to the application of these suicide genes, the increased understanding of the molecular mechanisms regulating adult neurogenesis allows investigators to develop new techniques to block neurogenesis (Mu et al., 2010). For example, blockade of several important signaling cascades responsible for the proliferation or maintenance of NSCs, such as Notch (Breunig et al., 2007; Ables et al., 2010; Ehm et al., 2010; Imayoshi et al., 2010), Shh (Lai et al., 2003; Balordi and Fishell, 2007), Wnt (Lie et al., 2005), and BMP (Lim et al., 2000; Mira et al., 2010) signaling can manipulate the behavior of NSCs. Incomplete maintenance and premature neuronal differentiation will deplete the neural stem pool and, consequently, reduce the supply of new neurons. Conversely, increased stem cell maintenance at the expense of proper neuronal differentiation will impair the ability of NSCs to generate a sufficient number of new neurons.

As most of the available genetic methods aim to reduce neurogenesis, it is important to develop genetic techniques that are able to increase the magnitude of neurogenesis. One important question in the adult neurogenesis research field is why new neurons are supplied only to the $\mathrm{OB}$ and hippocampal DG. To address this issue, genetic methods that can forcibly induce neurogenesis in ectopic brain regions, such as the cerebral cortex, are hoped for.

There is growing evidence that adult neurogenesis intensely contributes to neural circuit plasticity (Deng et al., 2010). Adultborn DG granule cells exhibit stronger synaptic plasticity than mature granule cells, as indicated by their lower threshold for the induction of long-term potentiation (LTP) and their higher LTP amplitude (Schmidt-Hieber et al., 2004; Ge et al., 2007). LTP forma- tion between centrifugal projections from the piriform cortex and adult-born granule cells in the $\mathrm{OB}$ was reported. Interestingly, the synaptic plasticity of young newborn neurons in the OB occurs during a narrow time-window when new neurons are initially added to the circuit (Gao and Strowbridge, 2009; Nissant et al., 2009). The transgenic expression of genetically encoded tools that can modulate LTP formation, such as constitutively active CREB or dominant negative GluR1, may shed light on the functional significance of neuron addition on neural circuit plasticity.

Several technologies have been recently developed to control the activity of genetically specified neural populations. Chemically triggered activating or silencing genetic technologies include RASSLs (Alexander et al., 2009), MISTs (Karpova et al., 2005), AlstR (Tan et al., 2006), GluClab (Lerchner et al., 2007), TRPV1 (Arenkiel et al., 2008), and modified GABA-A receptors (Wulff et al., 2007). Optogenetic tools that allow for the fast stimulation and inhibition of genetically targeted neuronal populations on the millisecond timescale have recently been developed. The light-gated cation channel Channelrhodopsin-2 (ChR2) and the light-driven chloride ions pump halorhodopsin are powerful and versatile tools for controlling neuronal activity (Zhang et al., 2007). The feasibility of using optogenetics to precisely control the activity of adult-born OB interneurons has been recently reported (Bardy et al., 2010). These technical advances in the fine manipulation of adult-born neurons will contribute to unveil the functional significance of adult neurogenesis.

\section{COMBINATIONAL USE OF OTHER SSRS AND THE TET SYSTEM WITH THE Cre/LoxP SYSTEM}

In addition to the Cre/loxP system, other SSR systems, such as the Flp/FRT, PhiC31/att, and Dre/rox systems, have been used as powerful tools for genome manipulation in mice (Branda and Dymecki, 2004). The Cre/loxP system is most widely used to manipulate the 
mouse genome in vivo, because it is more efficient than the other SSR systems, although recent studies reported that codon-optimized versions of SSRs (e.g., Flpo, PhiC31o, and codon-improved Dre) had similar recombination efficiencies as Cre (Raymond and Soriano, 2007; Anastassiadis et al., 2009). Similar to the Cre/loxP system, these SSRs are able to induce intra-molecular recombination between their recognition sites when oriented in the same direction in cis, including the deletion of the flanked sequence (Raymond and Soriano, 2007). As Cre, Flp, PhiC31, and Dre recognize different target sequences, the combined use of these recombinases would facilitate more sophisticated genetic manipulation of restricted cell populations.

For example, intersectional genetic strategies using Cre and Flp have been reported (Figure 3; Awatramani et al., 2003; Farago et al., 2006; Miyoshi and Fishell, 2006; Sousa et al., 2009). In the Rosa26 knock-in intersectional reporter mice (e.g., R26::FLAP, RC::PFwe, and RCE:dual), dual recombinase responsive indicator alleles were designed, that express marker proteins only when Cre and Flp have been expressed in the same cell. As the majority of genes in the developing nervous system are expressed in multiple cell populations, these intersectional approaches provide important means to reduce complexity, such that smaller subpopulations of genetically defined cells can be targeted.

As the majority of adult NSC-specific genes are expressed in the SVZ of the lateral ventricle and the SGZ of the DG, most CreER driver strains (e.g., Nestin-CreER and GLAST-CreER) induce recombination in both NSCs. In order to selectively manipulate NSCs either in the SVZ or the SGZ, Cre- and Flp-mediated intersectional strategies will be useful. The increased understanding of the molecular mechanisms regulating adult neurogenesis will identify candidate genes for Flp-expressing transgenic mice. Several members of the basic helix-loop-helix (bHLH) or homeobox gene families are selectively expressed in precursors or stem cells in the germinal zone of the adult brain. Alternatively, regulatory elements of the genes responsible for neurotransmitter identity (e.g., glutamatergic for the DG and GABAergic for the $\mathrm{OB}$ ) could be attractive candidates for Flp-expressing transgenic mice.

Recently the Dymecki group reported an elegant strategy applying a Cre- and Flp-mediated intersectional approach to the functional investigation of genetically defined neuronal populations

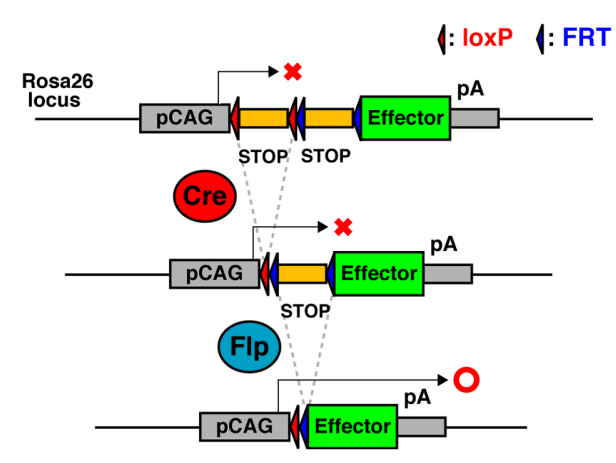

FIGURE 3 | Schematic of Cre- and Flp-dual recombinase responsive allele. The effector-encoding sequence is interrupted by a loxP-flanked STOP cassette followed by a FRT-flanked STOP cassette. The effector protein expresses only when Cre and Flp have been expressed in the same cell.
(Figure 3; Kim et al., 2009). RC::PFtox mice express the tetanus toxin light chain (tox), an inhibitor of vesicular neurotransmission, only when Cre and Flp have been expressed in the same cell. The development of intersectional transgenic mice with various effectors, for example, neurotoxins, optogenetic tools, neuronal activity modulators, etc., will contribute to the more sophisticated manipulation of newly born neurons in the adult brain.

The tetracycline-controlled transactivator (tTA)-dependent systems (Gossen and Bujard, 1992) have been used for inducible gene expression in adult NSCs. tTA is a fusion protein between the tetracycline repressor from the Tn 10 tetracycline resistance operon of Escherichia coli and the C-terminal domain of the VP16 transcription factor from HSV. The resulting hybrid transcriptional activator can trigger expression from a cognate promoter made of minimal promoter sequences placed downstream from multiple repeats of the tetracycline operator (tetO). tTA is constitutively active but its activity can be blocked by tetracycline or analogs such as Dox. The reverse tTA (rtTA), obtained by random mutagenesis of tTA, has the opposite features of tTA. It is constitutively silent but needs Dox to bind to the tet $\mathrm{O}$ sequences and induce gene transcription. Combined with tissue-specific promoters, the tetracycline-inducible system is able to provide transgenic mice with inducible, reversible, and spatially controlled transgene expression. As conditional expression systems with SSRs depend on the permanent intra-molecular recombination between their recognition sites, including the deletion of the flanked sequence, transgene expression is basically irreversible. The combinational use of the Tet system with the SSRs-mediated conditional expression systems will achieve the reversible manipulation of newly born neurons.

\section{ELECTROPORATION IN THE POSTNATAL BRAIN}

Although the use of transgenic or gene targeted mice has proved to be a powerful strategy for the manipulation of adult neurogenesis, we often suffer from the fact that transgenesis via oocyte injection or gene knock-in by homologous recombination in embryonic stem cells are time consuming and expensive. Gene transfer through the injection of plasmid DNA into embryos followed by electric pulses (electroporation) has developed into an important tool for functional analyses in vivo (Matsuda and Cepko, 2004; Shimogori and Ogawa, 2008). In utero electroporation in rodents is widely used, and more recently, gene transfer into postnatal neural tissues, including the postnatal and adult SVZ have been demonstrated (Barnabé-Heider et al., 2008; Boutin et al., 2008; Chesler et al., 2008). The lateral ventricle is readily accessible through minimally invasive techniques during the neonatal period. After electroporation, injected plasmid DNAs are incorporated into radial glial cells that populate the neonatal SVZ and send processes along the lateral ventricle. Electroporated radial glial cells differentiate into ependymal cells and SVZ astrocytes during the neonatal period. A subset of SVZ astrocytes generate transit-amplifying cells and neuroblasts, which migrate to the OB. Therefore, neonatal electroporation enables the visualization or manipulation of early postnatal neurogenesis. However, there is an important limitation of neonatal electroporation, plasmid dilution over the course of successive cell divisions results in lower and undetectable expression levels of transfected genes after several weeks of electroporation (Lacar et al., 2010). The use of vectors encoding transposons 
will permit genetic integration and the permanent expression of transfected genes in adult NSCs (Sato et al., 2007). Alternatively, electroporation of SSR-containing plasmids into the neonatal brain of transgenic mice with SSR-specific STOP cassettes, will achieve the prolonged expression of the gene of interest in adult NSCs and newborn neurons. An alternative approach is the electroporation of adult mice (Barnabé-Heider et al., 2008); however, this approach is technically more challenging and more damaging to the animals since it requires the use of a stereotactic frame.

Although we focused mainly on the transgenic or gene targeting approaches for the visualization and manipulation of adult neurogenesis in this review, virus vectors can also mediate efficient gene transfer in adult NSCs and newly born neurons (Consiglio et al., 2004).

\section{REFERENCES}

Ables, J. L., Decarolis, N. A., Johnson, M. A., Rivera, P. D., Gao, Z., Cooper, D. C., Radtke, F., Hsieh, J., and Eisch, A. J. (2010). Notchl is required for maintenance of the reservoir of adult hippocampal stem cells. J. Neurosci.30, 10484-10492.

Adam, Y., and Mizrahi, A. (2010). Circuit formation and maintenance - perspectives from the mammalian olfactory bulb. Curr. Opin. Neurobiol. 20, 134-140.

Ahn, S., and Joyner, A. L. (2005). In vivo analysis of quiescent adult neural stem cells responding to sonic hedgehog. Nature 437, 894-897.

Alexander, G. M., Rogan, S. C., Abbas, A. I., Armbruster, B. N., Pei, Y., Allen, J. A., Nonneman, R. J., Hartmann, J., Moy, S. S., Nicolelis, M. A., McNamara, J. O., and Roth, B. L. (2009). Remote control of neuronal activity in transgenic mice expressing evolved $\mathrm{G}$ protein-coupled receptors. Neuron 63, 27-39.

Altman, J. (1966). Autoradiographic and histological studies of postnatal neurogenesis. II. A longitudinal investigation of the kinetics, migration and transformation of cells incorporating tritiated thymidine in infant rats, with special reference to postnatal neurogenesis in some brain regions. J. Comp. Neurol. 1966, 431-474.

Altman, J. (1969). Autoradiographic and histological studies of postnatal neurogenesis. IV. Cell proliferation and migration in the anterior forebrain, with special reference to persisting neurogenesis in the olfactory bulb. $J$. Comp. Neurol. 137, 433-457.

Altman, J., and Das, G. D. (1965). Autoradiographic and histological evidence of postnatal hippocampal neurogenesis in rats. J. Comp. Neurol. 124, 319-335.

Alvarez-Buylla, A., Garcia-Verdugo, J. M., and Tramontin, A. D. (2001). A unified hypothesis on the lineage of neural stem cells. Nat. Rev. Neurosci. 2, 287-293.

Anastassiadis, K., Fu, J., Patsch, C., Hu, S. Weidlich, S., Duerschke, K., Buchholz, F., Edenhofer, F., and Stewart, A. F. (2009). Dre recombinase, like Cre, is a highly efficient site-specific recombinase in E. coli, mammalian cells and mice. Dis. Model. Mech. 2, 508-515.

Arenkiel, B. R., Klein, M. E., Davison, I. G., Katz, L. C., and Ehlers, M. D. (2008). Genetic control of neuronal activity in mice conditionally expressing TRPV1. Nat. Methods 5, 299-302.

Awatramani, R., Soriano, P., Mai, J. J., and Dymecki, S. (2001). An Flp indicator mouse expressing alkaline phosphatase from the ROSA26 locus. Nat. Genet. 29, 257-259.

Awatramani, R., Soriano, P., Rodriguez, C., Mai, J. J., and Dymecki, S. M. (2003). Cryptic boundaries in roof plate and choroid plexus identified by intersectional gene activation. Nat. Genet. 35 , 70-75.

Balordi, F., and Fishell, G. (2007). Mosaic removal of hedgehog signaling in the adult SVZ reveals that the residual wild-type stem cells have a limited capacity for self-renewal. J. Neurosci. 27, 4248-4259.

Bardy, C., Alonso, M., Bouthour, W., and Lledo, P. M. (2010). How, when, and where new inhibitory neurons release neurotransmitters in the adult olfactory bulb. J. Neurosci.30, 17023-17034.

Barnabé-Heider, F., Meletis, K., Eriksson, M., Bergmann, O., Sabelström, H., Harvey, M.A., Mikkers, H., and Frisén, J. (2008). Genetic manipulation of adult mouse neurogenic niches by in vivo electroporation. Nat. Methods 5, 89-96.

Batista-Brito, R., Close, J., Machold, R., and Fishell, G. (2008). The distinct temporal origins of olfactory bulb interneuron subtypes. J. Neurosci. 28, 3966-3975.

Boutin, C., Diestel, S., Desoeuvre, A., Tiveron, M. C., and Cremer, H. (2008).

\section{CONCLUSION}

Since the discovery of adult neurogenesis, many extensive studies have been performed on various aspects of adult neurogenesis. Postdevelopmental neurogenesis is found to be an evolutionarily conserved phenomenon, and functional importance on brain activities has just begun to be unveiled. Further understanding of adult neurogenesis will lead to the development of novel therapies for functional recovery after disease, trauma, or pathological aging.

\section{ACKNOWLEDGMENTS}

This work was supported by the Grants-in-aid from the Ministry of Education, Culture, Sports, Science and Technology of Japan. Itaru Imayoshi was supported by JST PRESTO program.

Efficient in vivo electroporation of the postnatal rodent forebrain. PLoS ONE 3, e1883. doi: 10.1371/journal. pone. 0001883

Branda, C. S., and Dymecki, S. M. (2004) Talking about a revolution: the impact of site-specific recombinases on genetic analysis in mice. Dev. Cell 6, 7-28.

Breunig, J. J., Silbereis, J., Vaccarino, F. M., Sestan, N., and Rakic, P. (2007). Notch regulates cell fate and dendrite morphology of newborn neurons in the postnatal dentate gyrus. Proc. Natl. Acad. Sci. U.S.A. 104 20558-20563.

Brockschnieder, D., Pechmann, Y. Sonnenberg-Riethmacher, E., and Riethmacher, D. (2006). An improved mouse line for Cre-induced cell ablation due to diphtheria toxin $\mathrm{A}$ expressed from the Rosa26 locus. Genesis 44, 322-327.

Buch, T., Heppner, F. L., Tertilt, C., Heinen, T. J., Kremer, M., Wunderlich, F. T., Jung, S., and Waisman, A. (2005). A Cre-inducible diphtheria toxin receptor mediates cell lineage ablation after toxin administration. Nat. Methods 2 , 419-426.

Burns, K. A., Ayoub, A. E., Breunig, J. J., Adhami, F., Weng, W. L., Colbert, M. C., Rakic, P., and Kuan, C. Y. (2007). Nestin-CreER mice reveal DNA synthesis by nonapoptotic neurons following cerebral ischemia hypoxia. Cereb. Cortex 17, 2585-2592.

Carlén, M., Meletis, K., Barnabé-Heider F., and Frisén, J. (2006). Genetic visualization of neurogenesis. Exp. Cell Res 312, 2851-2859.

Carleton, A., Petreanu, L. T., Lansford, R., Alvarez-Buylla, A., and Lledo, P. M. (2003). Becoming a new neuron in the adult olfactory bulb. Nat. Neurosci. 6 , 507-518.

Casanova, E., Fehsenfeld, S., Lemberger, T., Shimshek, D. R., Sprengel, R., and Mantamadiotis, T. (2002). ER-based double iCre fusion protein allows partial recombination in forebrain. Genesis 34, 208-214.

Chen, J., Kwon, C. H., Lin, L., Li, Y., and Parada, L. F. (2009). Inducible sitespecific recombination in neural stem/progenitor cells. Genesis 47, 122-131.

Cheng, X., Li, Y., Huang, Y., Feng, X., Feng, G., and Xiong,Z.Q. (2010). Pulse labeling and long-term tracing of newborn neurons in the adult subgranular zone. Cell Res. 12, 338-349.

Chesler, A. T., Le Pichon, C. E., Brann, J. H., Araneda, R. C., Zou, D. J., and Firestein, S. (2008). Selective gene expression by postnatal electroporation during olfactory interneuron neurogenesis. PLoS ONE 3, e1517. doi: 10.1371/journal.pone.0001517

Chow, L. M., Zhang, J., and Baker, S. J. (2008). Inducible Cre recombinase activity in mouse mature astrocytes and adult neural precursor cells. Transgenic Res. 17, 919-928.

Consiglio, A., Gritti, A., Dolcetta, D., Follenzi, A., Bordignon, C., Gage, F. H., Vescovi, A. L., and Naldini, L. (2004). Robust in vivo gene transfer into adult mammalian neural stem cells by lentiviral vectors. Proc. Natl. Acad. Sci. U.S.A. 101, 14835-14840.

Deng, W., Aimone, J. B., and Gage, F. H. (2010). New neurons and new memories: how does adult hippocampal neurogenesis affect learning and memory? Nat. Rev. Neurosci. 11, 339-350.

Deng, W., Saxe, M. D., Gallina, I. S., and Gage, F.H. (2009). Adult-born hippocampal dentate granule cells undergoing maturation modulate learning and memory in the brain. J. Neurosci. 29, 13532-13542.

Doetsch, F., Caille, I., Lim, D. A., GarciaVerdugo, J. M., and Alvarez-Buylla, A. (1999).Subventricular zone astrocytes are neural stem cells in the adult mammalian brain. Cell 97, 703-716.

Doetsch, F., Garcia-Verdugo, J. M., and Alvarez-Buylla, A. (1997). Cellular composition and three-dimensional 
organization of the subventricular germinal zone in the adult mammalian brain. J. Neurosci. 17, 5046-5061.

Dupret, D., Revest, J. M., Koehl, M., Ichas, F., De Giorgi, F., Costet, P., Abrous, D. N., and Piazza, P. V. (2008). Spatial relational memory requires hippocampal adult neurogenesis. PLoS ONE 3, e1959. doi:10.1371/journal. pone.0001959

Ehm, O., Göritz, C., Covic, M., Schaffner, I., Schwarz, T. J., Karaca, E., Kempkes, B., Kremmer, E., Pfrieger, F. W., Espinosa, L., Bigas, A., Giachino, C., Taylor, V., Frisén, J., and Lie, D. C. (2010). RBPJkappa-dependent signaling is essential for long-term maintenance of neural stem cells in the adult hippocampus. J. Neurosci. 30, 13794-13807.

Eriksson, P. S., Perfililieva, E., BjorkEriksson, T., Alborn, A. M., Nordborg, C., Peterson, D. A., and Gage, F. H. (1998). Neurogenesis in the adult human hippocampus. Nat. Med. 4, 1313-1317.

Farago, A. F., Awatramani, R. B., and Dymecki, S. M. (2006). Assembly of the brainstem cochlear nuclear complex is revealed by intersectional and subtractive genetic fate maps. Neuron 20, 205-218.

Favaro, R., Valotta, M., Ferri,A. L., Latorre, E., Mariani, J., Giachino, C., Lancini, C., Tosetti, V., Ottolenghi, S., Taylor, V., and Nicolis, S. K. (2009). Hippocampal development and neural stem cell maintenance require Sox2-dependent regulation of Shh. Nat. Neurosci. 12, 1248-1256.

Feil, R., Wagner, J., Metzger. D., and Chambon, P. (1997). Regulation of Cre recombinase activity by mutated estrogen receptor ligand-binding domains. Biochem. Biophys. Res. Commun. 237, 752-757.

Gage, F. H. (2000). Mammalian neural stem cells. Science 287, 1433-1438.

Ganat, Y. M., Silbereis, J., Cave, C., Ngu, H., Anderson, G. M., Ohkubo, Y., Ment, L. R., and Vaccarino, F. M. (2006). Early postnatal astroglial cells produce multilineage precursors and neural stem cells in vivo. J. Neurosci. 26, 8609-8621.

Gao, Y., and Strowbridge, B. W. (2009). Long-term plasticity of excitatory inputs to granule cells in the rat olfactory bulb. Nat. Neurosci. 12, 731-733.

Garcia, A. D., Doan, N. B., Imura, T., Bush, T. G., and Sofroniew, M. V. (2004). GFAP-expressing progenitors are the principal source of constitutive neurogenesis in adult mouse forebrain. Nat. Neurosci. 7, 1233-1241.

Garcia, A. D., Petrova, R., Eng, L., and Joyner, A. L. (2010). Sonic hedgehog regulates discrete populations of astro- cytes in the adult mouse forebrain. $J$. Neurosci. 30, 13597-13608.

Ge, S., Yang, C. H., Hsu, K. S., Ming, G. L., and Song, H. (2007). A critical period for enhanced synaptic plasticity in newly generated neurons of the adult brain. Neuron 54, 559-566.

Giachino, C., and Taylor, V. (2009). Lineage analysis of quiescent regenerative stem cells in the adult brain by genetic labelling reveals spatially restricted neurogenic niches in the olfactory bulb. Eur. J. Neurosci. 30, 9-24.

Gossen, M., and Bujard, H. (1992). Tight control of gene expression in mammalian cells by tetracycline-responsive promoters. Proc. Natl. Acad. Sci. U.S.A. $89,5547-5551$.

Gould, E. (2007). How widespread is adult neurogenesis in mammals? Nat. Rev. Neurosci. 8, 481-488.

Hippenmeyer, S., Vrieseling, E., Sigrist, M., Portmann, T., Laengle, C., Ladle, D. R., and Arber, S. (2005). A developmental switch in the response of DRG neurons to ETS transcription factor signaling. PLoS Biol. 3, e159. doi:10.1371/ journal.pbio.0030159

Imayoshi, I., Ohtsuka, T., Metzger, D., Chambon, P., and Kageyama, R. (2006). Temporal regulation of Cre recombinase activity in neural stem cells. Genesis 44, 233-238.

Imayoshi, I., Sakamoto, M., Ohtsuka, T., and Kageyama, R. (2009). Continuous neurogenesis in the adult brain. Dev. Growth Differ. 51, 379-386.

Imayoshi, I., Sakamoto, M., Ohtsuka, T., Takao, K., Miyakawa, T., Yamaguchi, M., Mori, K., Ikeda, T., Itohara, S., and Kageyama, R. (2008). Roles of continuous neurogenesis in the structural and functional integrity of the adult forebrain. Nat. Neurosci. 11, 1153-1161.

Imayoshi, I., Sakamoto, M., Yamaguchi, M., Mori, K., and Kageyama, R. (2010). Essential roles of Notch signaling in maintenance of neural stem cells in developing and adult brains. $J$. Neurosci. 30, 3489-3498.

Indra, A. K., Li, M., Brocard, J., Warot, X., Bornert, J. M., Gérard, C., Messaddeq, N., Chambon, P., and Metzger, D. (2000). Targeted somatic mutagenesis in mouse epidermis. Horm. Res. 54, 296-300.

Kaplan, M. S., and Hinds, J. W. (1977). Neurogenesis in the adult rat: electron microscopic analysis of light radioautographs. Science 197, 1092-1094.

Karpova, A. Y., Tervo, D. G., Gray, N. W., and Svoboda, K. (2005). Rapid and reversible chemical inactivation of synaptic transmission in genetically targeted neurons. Neuron 48 , 727-735.
Kawamoto, S., Niwa, H., Tashiro, F., Sano, S., Kondoh, G., Takeda, J., Tabayashi, K., and Miyazaki, J. (2000). A novel reporter mouse strain that expresses enhanced green fluorescent protein upon Cre-mediated recombination. FEBS Lett. 470, 263-268.

Kee, N., Teixeira, C. M., Wang, A. H., and Frankland, P. W. (2007). Preferential incorporation of adult-generated granule cells into spatial memory networks in the dentate gyrus. Nat. Neurosci. 10, 355-362.

Kelsch, W., Lin, C. W., and Lois, C. (2008) Sequential development of synapses in dendritic domains during adult neurogenesis. Proc. Natl. Acad. Sci. U.S.A. 105, 16803-16808.

Kelsch, W., Lin, C. W., Mosley, C. P., and Lois, C. (2009). A critical period for activity-dependent synaptic development during olfactory bulb adult neurogenesis. J. Neurosci. 29, 11852-11858.

Kelsch, W., Sim, S., and Lois, C. (2010). Watching synaptogenesis in the adult brain. Annu. Rev. Neurosci. 33, 131-149.

Kim, E. J., Leung, C. T., Reed, R. R., and Johnson, J.E. (2007). In vivo analysis of Ascll defined progenitors reveals distinct developmental dynamics during adult neurogenesis and gliogenesis. J. Neurosci. 27, 12764-12774.

Kim, J. C., Cook, M. N., Carey, M. R., Shen, C., Regehr, W. G., and Dymecki, S. M. (2009). Linking genetically defined neurons to behavior through a broadly applicable silencing allele. Neuron 63 305-315.

Kriegstein, A., and Alvarez-Buylla, A (2009). The glial nature of embryonic and adult neural stem cells. Annu. Rev Neurosci. 32, 149-184.

Kuo, C. T., Mirzadeh, Z., SorianoNavarro, M., Rasin, M., Wang, D., Shen, J., Sestan, N., Garcia-Verdugo, J., Alvarez-Buylla, A., Jan, L. Y., and Jan, Y. N. (2006). Postnatal deletion of Numb/Numblike reveals repair and remodeling capacity in the subventricular neurogenic niche. Cell 127, 1253-1264.

Lacar, B., Young, S. Z., Platel, J. C., and Bordey, A. (2010). Imaging and recording subventricular zone progenitor cells in live tissue of postnatal mice. Front. Neurosci. 4:43. doi:10.3389/fnins.2010.00043

Lagace, D. C., Whitman, M. C., Noonan, M. A., Ables, J. L., DeCarolis, N. A., Arguello, A. A., Donovan, M. H., Fischer, S. J., Farnbauch, L. A., Beech, R. D., DiLeone, R. J., Greer, C. A., Mandyam, C. D., and Eisch, A. J. (2007). Dynamic contribution of nestin-expressing stem cells to adult neurogenesis. J. Neurosci. 27, 2623-2629.
Lai, K., Kaspar, B. K., Gage, F. H., and Schaffer, D. V. (2003). Sonic hedgehog regulates adult neural progenitor proliferation in vitro and in vivo. Nat. Neurosci. 6, 21-27.

Lazarini, F., and Lledo, P. M. (2011). Is adult neurogenesis essential for olfaction? Trends Neurosci. 34, 20-30.

Lendahl, U., Zimmerman, L. B., and McKay, R. D. G. (1990). CNS stem cells express a new class of intermediate filament protein. Cell 60, 585-595.

Lerchner, W., Xiao, C., Nashmi, R., Slimko, E. M., van Trigt, L., Lester, H. A., and Anderson, D. J. (2007). Reversible silencing of neuronal excitability in behaving mice by a genetically targeted, ivermectin-gated $\mathrm{Cl}$ - channel. Neuron 54, 35-49.

Li, M., Indra, A. K., Warot, X., Brocard, J., Messaddeq, N., Kato, S., Metzger, D., and Chambon, P. (2000). Skin abnormalities generated by temporally controlled RXRalpha mutations in mouse epidermis. Nature 407, 633-636.

Lie, D. C., Colamarino, S. A., Song, H. J., Désiré, L., Mira, H., Consiglio, A., Lein, E. S., Jessberger, S., Lansford, H., Dearie, A. R., and Gage, F. H. (2005). Wnt signalling regulates adult hippocampal neurogenesis. Nature 437, 1370-1375.

Lim, D. A., Tramontin, A. D., Trevejo, J. M., Herrera, D. G., García-Verdugo, J. M., and Alvarez-Buylla, A. (2000). Noggin antagonizes BMP signaling to create a niche for adult neurogenesis. Neuron 28, 713-726.

Liu, H. K., Belz, T., Bock, D., Takacs, A., Wu, H., Lichter, P., Chai, M., and Schutz, G. (2008). The nuclear receptor tailless is required for neurogenesis in the adult subventricular zone. Genes Dev. 22, 2473-2478.

Livneh, Y., Feinstein, N., Klein, M., and Mizrahi, A. (2009). Sensory input enhances synaptogenesis of adultborn neurons. J. Neurosci. 29, 86-97.

Lledo, P. M., Alonso, M., and Grubb, M.S. (2006). Adult neurogenesis and functional plasticity in neuronal circuits. Nat. Rev. Neurosci. 7, 179-193.

Lobe, C. G., Koop, K. E., Kreppner, W., Lomeli, H., Gertsenstein, M., and Nagy, A. (1999). Z/AP, a double reporter for cre-mediated recombination. Dev. Biol. 208, 281-292.

Lugert, S., Basak, O., Knuckles, P., Haussler, U., Fabel, K., Goetz, M., Haas, C. A., Kempermann, G., Taylor, V., and Giachino, C. (2010). Quiescent and active hippocampal neural stem cells with distinct morphologies respond selectively to physiological and pathological stimuli and aging. Cell Stem Cell 6,445-456.

Luo, L., Callaway, E. M., and Svoboda, K. (2008). Genetic dissection of neural circuits. Neuron 57, 634-660. 
Madisen, L., Zwingman, T. A., Sunkin, S. M., Oh, S. W., Zariwala, H. A., Gu, H., Ng, L. L., Palmiter, R. D., Hawrylycz, M. J., Jones, A. R., Lein, E. S., and Zeng, H. (2010). A robust and high-throughput Cre reporting and characterization system for the whole mouse brain. Nat. Neurosci. 13, 133-140.

Mao, X., Fujiwara, Y., Chapdelaine, A., Yang, H., and Orkin, S. H. (2001). Activation of EGFP expression by Cremediated excision in a new ROSA26 reporter mouse strain. Blood 97, 324-326.

Matsuda, T., and Cepko, C. L. (2004). Electroporation and RNA interference in the rodent retina in vivo and in vitro. Proc. Natl. Acad. Sci. U.S.A. 101, 16-22.

Matsuda, T., and Cepko, C. L. (2007). Controlled expression of transgenes introduced by in vivo electroporation. Proc. Natl. Acad. Sci. U.S.A. 104, 1027-1032.

McKay, R. (1997). Stem cell in the central nervous system. Science 276, 66-71.

Metzger, D., Clifford, J., Chiba, H., and Chambon, P. (1995). Conditional site-specific recombination in mammalian cells using a ligand-dependent chimeric Cre recombinase. Proc. Natl. Acad. Sci. U.S.A. 92, 6991-6995.

Mignone, J. L., Kukekov, V., Chiang, A., Steindler, D., and Enikolopov, G. (2004). Neural stem and progenitor cells in nestin-GFP transgenic mice. J. Comp. Neurol. 469, 311-324.

Ming, G., and Song, H. J. (2005). Adult neurogenesis in the mammalian central nervous system. Annu. Rev. Neurosci. 28, 223-250.

Mira, H., Andreu, Z., Suh, H., Lie, D. C., Jessberger, S., Consiglio, A., San Emeterio, J., Hortigüela, R., MarquésTorrejón, M. A., Nakashima, K., Colak, D., Götz, M., Fariñas, I., and Gage, F. H. (2010). Signaling through BMPR-IA regulates quiescence and long-term activity of neural stem cells in the adult hippocampus. Cell Stem Cell 7, 78-89.

Miyamichi, K., Amat, F., Moussavi, F., Wang, C., Wickersham, I., Wall, N. R., Taniguchi, H., Tasic, B., Huang, Z. J., He, Z., Callaway, E. M., Horowitz, M. A., and Luo, L. (2011). Cortical representations of olfactory input by transsynaptic tracing. Nature 472, 191-196.

Miyoshi, G., and Fishell, G. (2006). Directing neuron-specific transgene expression in the mouse CNS. Curr. Opin. Neurobiol. 16, 577-584.

Mori, T., Tanaka, K., Buffo, A., Wurst, W., Kühn, R., and Götz, M. (2006). Inducible gene deletion in astroglia and radial glia - a valuable tool for functional and lineage analysis. Glia 54, 21-34.
Mu, Y., Lee, S. W., and Gage, F. H. (2010). Signaling in adult neurogenesis. Curr. Opin. Neurobiol. 20, 416-423.

Muzumdar, M. D., Tasic, B., Miyamichi, K., Li, L., and Luo, L. (2007). A global double-fluorescent Cre reporter mouse. Genesis 45, 593-605.

Nagy, A. (2000). Cre recombinase: the universal reagent for genome tailoring. Genesis 26, 99-109.

Ninkovic,J.,Mori, T., and Götz, M. (2007). Distinct modes of neuron addition in adult mouse neurogenesis. J. Neurosci. 27, 10906-10911.

Nissant, A., Bardy, C., Katagiri, H., Murray, K., and Lledo, P. M. (2009). Adult neurogenesis promotes synaptic plasticity in the olfactory bulb. Nat. Neurosci. 12, 728-730.

Novak, A., Guo, C., Yang, W., Nagy, A., and Lobe, C. G. (2000). Z/EG, a double reporter mouse line that expresses enhanced green fluorescent protein upon Cre-mediated excision. Genesis $28,147-155$.

Pastrana, E., Cheng, L. C., and Doetsch, F. (2009). Simultaneous prospective purification of adult subventricular zone neural stem cells and their progeny. Proc. Natl. Acad. Sci. U.S.A. 106, 6387-6392.

Possemato, R., Eggan, K., Moeller, B. J., Jaenisch, R., and Jackson-Grusby, L. (2002). Flp recombinase regulated lacZ expression at the ROSA26 locus. Genesis 32, 184-186.

Raymond, C. S., and Soriano, P. (2007). High-efficiency FLP and PhiC31 sitespecific recombination in mammalian cells. PLoS ONE 2, e162. doi:10.1371/ journal.pone. 0000162

Sato, Y., Kasai, T., Nakagawa, S., Tanabe, K., Watanabe, T., Kawakami, K., and Takahashi, Y. (2007). Stable integration and conditional expression of electroporated transgenes in chicken embryos. Dev. Biol. 305, 616-624.

Saxe, M. D., Battaglia, F., Wang, J. W., Malleret, G., David, D. J., Monckton, J. E., Garcia, A., D., Sofroniew, M. V., Kandel, E. R., Santarelli, L., Hen, R. and Drew, M. R. (2006). Ablation of hippocampal neurogenesis impairs contextual fear conditioning and synaptic plasticity in the dentate gyrus. Proc. Natl. Acad. Sci. U.S.A. 103, 17501-17506.

Schmidt-Hieber, C., Jonas, P., and Bischofberger, J. (2004). Enhanced synaptic plasticity in newly generated granule cells of the adult hippocampus. Nature 429, 184-187.

Seri, B., Garcia-Verdugo, J. M., ColladoMorente, L., McEwen, B. S., and Alvarez-Buylla, A. (2004). Cell types, lineage, and architecture of the germinal zone in the adult dentate gyrus. $J$. Comp. Neurol. 478, 359-378.
Shimogori, T., and Ogawa, M. (2008). Gene application with in utero electroporation in mouse embryonic brain. Dev. Growth Differ. 50 499-506.

Singer, B. H., Jutkiewicz, E. M., Fuller, C. L., Lichtenwalner, R. J., Zhang, H., Velander, A. J., Li, X., Gnegy, M. E., Burant, C. F., and Parent, J. M. (2009). Conditional ablation and recovery of forebrain neurogenesis in the mouse. J. Comp. Neurol. 514, 567-582.

Slezak, M., Göritz, C., Niemiec, A., Frisén, J., Chambon, P., Metzger, D. and Pfrieger, F. W. (2007). Transgenic mice for conditional gene manipulation in astroglial cells. Glia 55, 1565-1576.

Soriano, P. (1999). Generalized lacZ expression with the ROSA26 Cre reporter strain. Nat. Genet. 21 70-71.

Sousa, V. H., Miyoshi, G., HjerlingLeffler, J., Karayannis, T., and Fishell, G. (2009). Characterization of Nkx62-derived neocortical interneuron lineages. Cereb. Cortex 19 (Suppl. 1), i1-i10.

Srinivas, S., Watanabe, T., Lin, C. S., William, C. M., Tanabe, Y., Jessell, T. M., and Costantini, F. (2001). Cre reporter strains produced by targeted insertion of EYFP and ECFP into the ROSA26 locus. BMC Dev. Biol. 1, 4 doi:10.1186/1471-213X-1-4

Steiner, B., Klempin, F., Wang, L., Kott, M., Kettenmann, H., and Kempermann, G. (2006). Type-2 cells as link between glial and neuronal lineage in adult hippocampal neurogenesis. Glia 54 805-814.

Strathdee, D., Ibbotson, H., and Grant, S. G. (2006). Expression of transgenes targeted to the Gt(ROSA)26Sor locus is orientation dependent. PLOS ONE 1 , e4. doi:10.1371/journal.pone.0000004

Suh, H., Consiglio, A., Ray, J., Sawai, T., D-Amour, K. A., and Gage, F. H. (2007). In vivo fate analysis reveals the multipotent and self-renewal capacities of Sox $2+$ neural stem cells in the adult hippocampus. Cell Stem Cell 1, 515-528.

Tan, E. M., Yamaguchi, Y., Horwitz, G. D. Gosgnach, S., Lein, E. S., Goulding, M., Albright, T. D., and Callaway, E. M. (2006). Selective and quickly reversible inactivation of mammalian neurons in vivo using the Drosophila allatostatin receptor. Neuron 51 , 157-170.

Temple, S. (2001). The development of neural stem cells. Nature 414,112-117.

van Praag, H., Schinder, A. F., Christie, B. R., Toni, N., Palmer, T. D., and Gage, F. H. (2002). Functional neurogenesis in the adult hippocampus. Nature 415, 1030-1034
Vintersten, K., Monetti, C., Gertsenstein, M., Zhang, P., Laszlo, L., Biechele, S., and Nagy, A. (2004). Mouse in red: red fluorescent protein expression in mouse ES cells, embryos, and adult animals. Genesis 40, 241-246.

Wickersham, I. R., Lyon, D. C., Barnard, R. J., Mori, T., Finke, S., Conzelmann, K. K., Young, J.A., and Callaway, E. M. (2007) Monosynaptic restriction of transsynaptic tracing from single, genetically targeted neurons. Neuron 53, 639-647.

Wojtowicz, J. M. (2006). Irradiation as an experimental tool in studies of adult neurogenesis. Hippocampus 16, 261-266.

Wu, S., Ying, G., Wu, Q., and Capecchi, M. R. (2007). Toward simpler and faster genome-wide mutagenesis in mice. Nat. Genet. 39, 922-930.

Wulff, P., Goetz, T., Leppä, E., Linden, A. M., Renzi, M., Swinny, J. D. Vekovischeva, O. Y., Sieghart, W., Somogyi, P., Korpi, E. R., Farrant, M., and Wisden, W. (2007). From synapse to behavior: rapid modulation of defined neuronal types with engineered GABAA receptors. Nat. Neurosci. 10, 923-929.

Young, K. M., Mitsumori, T., Pringle, N., Grist, M., Kessaris, N., and Richardson, W. D. (2010). An Fgfr3-iCreER(T2) transgenic mouse line for studies of neural stem cells and astrocytes. Glia 58, 943-953.

Zambrowicz, B. P., Imamoto, A., Fiering, S., Herzenberg, L. A., Kerr, W. G., and Soriano, P. (1997). Disruption of overlapping transcripts in the ROSA beta geo 26 gene trap strain leads to widespread expression of betagalactosidase in mouse embryos and hematopoietic cells. Proc. Natl. Acad. Sci. U.S.A. 94, 3789-3794.

Zhang, J., Giesert, F., Kloos, K., Vogt Weisenhorn, D. M., Aigner, L., Wurst, W., and Couillard-Despres, S. (2010). A powerful transgenic tool for fate mapping and functional analysis of newly generated neurons. BMC Neurosci. 11,158. doi:10.1186/1471-2202-11-158

Zhang, F., Wang, L. P., Brauner, M., Liewald, J. F., Kay, K., Watzke, N., Wood, P. G., Bamberg, E., Nagel, G., Gottschalk, A., and Deisseroth, K. (2007). Multimodal fast optical interrogation of neural circuitry. Nature 446, 633-639.

Zhao, C., Teng, E. M., Summers, R. G. Jr., Ming, G. L., and Gage, F. H. (2006). Distinct morphological stages of dentate granule neuron maturation in the adult mouse hippocampus. $J$. Neurosci. 26, 3-11.

Zimmerman, L., Lendahl, U., Cunningham, M., McKay, R., Parr, B. Gavin, B., Mann, J., Vassileva, G., and 
McMahon, A. (1994). Independent regulatory elements in the nestin gene direct transgene expression to neural stem cells or muscle precursors. Neuron 12, 11-24.

Conflict of Interest Statement: The authors declare that the research was conducted in the absence of any commercial or financial relationships that could be construed as a potential conflict of interest.

Received: 31 January 2011; accepted: 19 April 2011; published online: 02 May 2011.
Citation: Imayoshi I, Sakamoto $M$ and Kageyama R (2011) Genetic methods to identify and manipulate newly born neurons in the adult brain. Front. Neurosci. 5:64. doi: 10.3389/fnins.2011.00064

This article was submitted to Frontiers in Neurogenesis, a specialty of Frontiers in Neuroscience.
Copyright $@ 2011$ Imayoshi, Sakamoto and Kageyama. This is an open-access article subject to a non-exclusive license between the authors and Frontiers Media SA, which permits use, distribution and reproduction in other forums, provided the original authors and source are credited and other Frontiers conditions are complied with. 